\title{
Formulation and evaluation of folic acid conjugated gliadin nanoparticles of curcumin for targeting colon cancer cells
}

\author{
Shipra Sonekar*, Manoj Kumar Mishra, Anil Kumar Patel, Suresh Kumar Nair, Chandra Shekhar Singh, \\ Amit Kumar Singh \\ Shambhunath Institute of Pharmacy, Jhalwa, Allahabad, Uttar Pradesh, India.
}

\section{ARTICLE INFO}

Article history:

Received on: 08/05/2016

Revised on: 12/07/2016

Accepted on: 30/07/2016

Available online: 29/10/2016

Key words:

Chlorella sp., Synechocystis

sp., nitrogen, phosphorus,

waste water.

\begin{abstract}
Preparation of folic acid (FA) conjugated (FA-CUR-GNPs) and non-conjugated (CUR-GNPs) gliadin nanoparticles of curcumin were successfully formulated by desolvation method for oral delivery of drug for targeting colon cancer cell. F1, F3, F5 (conjugated) and F2, F4, F6 (Non-conjugated) were formulated using various drug-polymer ratio (1:2). They were further characterized by FTIR, Mass spectroscopy, NMR, solubility studies, entrapment efficiency, TEM, particle size, surface charge, In-vitro release studies, In vivo toxicity studies and simultaneously evaluated. F3 (curcumin 10mg, gliadin 20mg and FA 5mg) and F4 (curcumin 10mg and gliadin 20mg) were found as the optimized formulation among both the categories. For F3 and F4 formulations; average particle size $(168.1$ and $195.7 \mathrm{~nm})$, zeta potential $(-16.5$ and $-24.4 \mathrm{mV})$, cumulative $\%$ drug release $(92.92$ and $94 \%$ ) and In vivo toxicity studies were conducted and compared with the control (phosphatebuffer saline, pH 6.8) reveals no toxicity. From the characterization and evaluation studies it was identified that F4 (FA-CUR-GNPs) had better solubility, In vitro release profile and no specified In-vivo toxicity than F3 (CUR-GNPs) formulation with nano-range particle size throughout the experiment. Improved bioavailability and increase targeting capacity toward colon cancer tumor cells were successfully achieved.
\end{abstract}

\section{INTRODUCTION}

Prolonged exposure to almost any provocative agent in the environment (radiation, chemicals, viruses, inflammation, etc.) can potentially cause cancer (Seyfried and Shelton, 2010). Nano therapeutics are the most advanced field which are reduced the limitations of conventional drug delivery system such as lack of specified targeting, lower aqueous solubility, poor oral bioavailability and decreased therapeutic indices (Gelperina et al., 2005). As per literature, folate receptor has been explored to deliver protein toxins, low-molecular weight chemotherapeutic agents, radio-imaging agents, MRI contrast agents, radio therapeutic agents, liposomes containing chemotherapeutic drugs, genes, antisense oligonucleotides, ribozymes, and immunotherapeutic agents to cancer cells (Duthie, 1999).

\footnotetext{
* Corresponding Author

Shipra Sonekar, Shambhunath Institute of Pharmacy, Jhalwa,

Allahabad, Uttar Pradesh, India. Email: shipra.qc123 @ gmail.com
}

The proliferancy of colon cancer is increasing day by day in developed countries (Pisani et al., 2002). Pre-clinical research studies using curcumin has proved reduction of various cancerous cells due to inhibition of NF- $\mathrm{KB}$ and $\mathrm{COX}-2$, arachidonic acid metabolism via lipoxygenase, scavenging of free radicals, expression of inflammatory cytokines IL1b, IL-6, TNF-a and down-regulation of enzymes (Jobin et al., 1999; Huang et al., 1991; Cho et al., 2007; Liu et al., 1993). Curcumin (CUR), is a bis-R,-unsaturated-diketone (commonly called diferuloylmethane) which exhibits keto-enol tautomerism having a predominant keto form in acidic and neutral solutions and stable enol form in alkaline medium is obtained from the rhizome of the perennial herb Curcuma longa, can reverse the multidrug resistance phenomenon (Bansal et al., 2011). It inhibits proliferation in various cancer cells isolated from malignancies like leukemia, breast, lung, prostate and colon tumors (Anand et al., 2008). Studies were also done in various tumerogenetic models (Tuttle et al., 2012). Curcumin blocks the NFkB pathway responsible for tumor cell survival and metastasis (Srimal and Dhawan, 1973). 
Folate receptor conjugate vitamin and drugs with a high affinity and carries these bound molecules into the tumor tissues (Xiang et al., 2008). Cellular uptake of folates are facilitated by a high affinity glycosylphosphatidylinositol-linked FR $(\mathrm{K} D \approx 100$ pM), which exhibits highly limited distribution. Gliadin (GNP) nanoparticles are suitable for both passive and active drug targeting offers a wide variety of possibilities for preparing better tolerated parenteral and oral formulations either maintaining the same characteristics engineered to pharmacokinetic and tissue distribution or enhancing the site specific delivery (Jahanshahi and Babaei, 2008).

Present study was to design and develop gliadin nanoparticles of curcumin for oral delivery of drug for targeting colon cancer cell.

\section{MATERIAL AND METHODS}

\section{Materials}

Curcumin, Gliadin, Pluronic F-68 and Folic Acid were obtained from Sigma Aldrich Corp., Switzerland as gift samples. Ethanol was obtained from Qualigens Fine Chemicals (P) Ltd, Mumbai, India; Chloroform, Dimethyl sulfoxide (DMSO), Ethylene Dichloride (EDC) and Dichloromethane were obtained from Merck Specialties Pvt. Ltd., New Delhi, India; Sodium hydrogen phosphate and Disodium Hydrogen Phosphate were obtained from Sarabhai M. Chemicals, Vadodara, India. Acetone and Benzene were obtained from Avantor Performance Materials Ltd., Gurgaon, India.

\section{Identification and characterization of curcumin}

\section{Fourier transform infrared spectroscopy (FTIR)}

The Infrared absorption spectrum of drug, polymer, folic acid conjugation (FA-CUR-GNP) and folic acid non- conjugation (CUR-GNP) were obtained by finely grounding and dispersing in $\mathrm{KBr}$ using FTIR spectroscopy was performed on a Spectrum 65 (Perkin Elmer). Spectra were recorded between 4000 and $600 \mathrm{~cm}^{-1}$ range. The IR absorption spectrum of drug, polymer, FA-CUR-GNP and CUR-GNP exhibits maxima only at the same wavelengths as that of a reference standard.

\section{Mass spectroscopy}

Electron Spray Ionization mass spectra (ESI-MS) of Curcumin and Gliadin were performed.

\section{NMR spectrum of curcumin}

${ }^{1} \mathrm{H}$ NMR spectra were taken on Bruker Advance DRX500, Brucker Biosciences Corporation, Billerica, MA, USA at 500 $\mathrm{MHz} \mathrm{CD}_{3} \mathrm{OD}$ was taken as solvent. Pure drug curcumin $(5 \mathrm{mg}$ ) was dissolved in $1 \mathrm{ml}$ of $\mathrm{CD}_{3} \mathrm{OD}$ and subjected to NMR analysis.

\section{Solubility studies}

\section{Solubility profile of curcumin}

The equilibrium solubility of CUR was determined at different solvents. Equivalent amount of the drug was added and shaken in separate flask containing different solvents using a mechanical shaker at room temperature $\left(25^{\circ} \mathrm{C}\right)$ for $24 \mathrm{~h}(\mathrm{n}=3)$. The samples were then centrifuged at $2000 \mathrm{rpm}$, filtered through membrane filter $(0.45 \mu \mathrm{m})$ and analysed by HPLC to quantify dissolved drug content. The solubility of the drugs in water, methanol, ethanol and acetone were checked by High Performance Liquid Chromatography (HPLC) (Yanyu et al., 2006).

\section{Solubility profile of gliadin}

Gliadin is a prolamine, which is soluble only in suitable ratios of aqueous and organic phases. To find its solubility in different concentrations, $1 \%$ of the gliadin solutions were prepared by using different ratios of the ethanol and water and solubility were determined with the absorbance value from UV spectrophotometer at $283 \mathrm{~nm}$.

\section{Partition coefficient}

The Partition coefficient between n-octanol/water was determined at room temperature $\left(30{ }^{\circ} \mathrm{C}\right) .10 \mathrm{ml}$ of n-octanol and 10 $\mathrm{ml}$ of distilled water was taken in a glass stoppers graduated tube and $5 \mathrm{mg}$ of accurately weighted drug was added, the mixture was then shaken with the help of mechanical shaker for $24 \mathrm{~h}$ at room temperature the mixture was then transferred to a separating funnel and allowed to equilibrate for $6 \mathrm{~h}$. The aqueous and octanol phase were separated and filtered through membrane and drug content in aqueous phase was analyzed by UV-Visible spectrophotometer.

\section{Entrapment efficiency}

Drug was loaded by adding the drug with the gliadin polymer in the $70 \%$ alcoholic mixture. Different ratios of drug and polymers were taken and different batches of nanoparticle were prepared.

Drug entrapment efficiency (Moradhaseli et al., 2013) was determined by dispersing the nanoparticle suspension in the $70 \%$ aqueous ethanol and drug was extracted after vigorous vortexing followed by centrifugation. The curcumin concentration in the supernatant was determined with the help of UV spectrophotometer (Perkin Elmer, USA) at $549 \mathrm{~nm}$ and measured with the help of the calibration curve describing the absorbance and concentration relationship.

\section{Formulation development}

\section{Folic acid conjugation method}

FA-CUR-GNP ${ }_{S}$ were prepared by three step method. Firstly, the phenolic group of CUR easily attached to the amino group of GNP forming stable CUR-NH $\mathrm{N}_{2}$ bond. Secondly, EDC was used to activate the carboxyl group of gliadin forming an intermediate EDC- carboxylate. Thirdly, activated carbonyl group react subsequently with the free amino group of folic acid resulting in the formation of FA-CUR-GNP.

For Folic acid conjugation method; Folic acid ( $5 \mathrm{mg}$ ) was dissolved in $20 \% \mathrm{w} / \mathrm{v}$ aqueous solution of sodium hydroxide 
(10ml) and the formed solution was dropped into $10 \mathrm{ml}$ of phosphate buffer suspension ( $\mathrm{pH} 7.4$ ) containing $20 \mathrm{mg}$ of gliadinNPs under oscillation for $30 \mathrm{~min}$.

Loading efficiency of FA in gliadin-NPs was determined by measuring the difference between the initially added FA amount and FA in the supernatant. The collected products were washed 3-4 times with deionized water, centrifuged at $20000 \mathrm{rpm}$ for $15 \mathrm{~min}$ at $4^{\circ} \mathrm{C}$, and freeze dried to obtain powders.

\section{Preparation methodology of nanoparticles}

Gliadin nanoparticles were prepared by simple desolvation method (Coester et al., 2000). This method includes preparation of aqueous phase $\mathrm{A}$ and non-aqueous phase $\mathrm{B}$. Phase$\mathrm{B}$ containing different concentrations of gliadin solubilized at $25^{\circ} \mathrm{C}$ in $20 \mathrm{ml}$ of $70 \%$ ethanol/water mixture.

Drug (curcumin) was added to this phase in required quantities. Phase-A contains $0.5 \%$ concentrations of surfactant PF68 in 40ml of triple distilled water. These two phases were filtered (Millipore $0.45 \mu$ ). Then phase-B is added dropwise with the help of the disposable syringe for $1 \mathrm{~h}$. The nanoparticle suspension was concentrated using Rota vapor and were further purified by centrifugation for $15 \mathrm{~min}$ at $20000 \mathrm{rpm}$ at $4^{\circ} \mathrm{C}$. The supernatant formed was removed and pellets were re-suspended in $2 \mathrm{ml}$ of triple distilled water. Finally lyophilized by using 5\% mannitol as a cry protectant.

\section{Optimization of folic acid conjugated and non-conjugated nanoparticle}

The optimization was done by Zetasizer Nano-ZS (Malvern Instruments Inc., UK) and the formulation with better particle size and zeta potential were selected for the study. The samples were loaded onto $1 \mathrm{~cm}^{2}$ cuvettes in a thermostat chamber and particle size were determined (Table 1).

\section{Characterization of nanoparticles}

\section{Particle morphology by TEM}

The characterization of FA-CUR-GNP ${ }_{S}$ and CUR-GNP were done by using transmission electron microscopy (TEM, JEOL 1011, Japan). A drop each of FA-CUR-GNP ${ }_{S}$ and CUR$\mathrm{GNP}_{\mathrm{S}}$ were suspended in triple distilled water placed onto a carbon-coated copper grid, forming a thin liquid film negatively stained with a drop of $1 \%(\mathrm{w} / \mathrm{v})$ phosphotungstic acid. Excessive volume of staining solution was removed by using filter paper and the sample was air-dried. These samples were visualized under TEM and photographed.

\section{Particle size measurements}

The particle size (Storp et al., 2012) of FA-CUR-GNP and CUR-GNP $\mathrm{S}_{\mathrm{S}}$ were measured by Zetasizer Nano-ZS 90 (Malvern Instruments Inc., UK).

\section{Surface charge (zeta potential) studies (Weber et al., 2000)}

In disperse systems, electrical charges are developed at the interface between the dispersed phase and the aqueous medium. Two most common mechanisms are the ionization of surface functional groups and the specific adsorption of ions. The electrical charge determines the interaction between the particles of the dispersed phase and physical stability of the system, particularly for those in the colloidal size range (emulsion).

For a system when the zeta potential is relatively high ( $25 \mathrm{mV}$ or more, absolute value) the repulsive forces exceed the attractive London forces and the system is deflocculated. On the other hand, when the zeta potential is low, the attractive forces exceed the repulsive forces and leads to flocculation. Thus, the magnitude of the zeta potential gives an indication of the potential stability of the colloidal system (Table 2).

\section{In-vitro release studies}

In-vitro drug release studies were carried out for FACUR-GNP $_{S}$ and CUR-GNP ${ }_{S}$ in colonic fluids of $\mathrm{pH} 6.8 \pm 0.5$ for $48 \mathrm{~h}$ at $37^{\circ} \mathrm{C}$. The release studies of drug from both conjugated and non-conjugated were performed using a US Pharmacopeia dissolution rate test apparatus (paddle apparatus, $100 \mathrm{rpm}, 37 \pm$ $\left.0.1^{\circ} \mathrm{C}\right)$.

Samples $(2 \mathrm{ml})$ were withdrawn at appropriate time intervals and estimation of curcumin was done using UV spectrophotometer at $424 \mathrm{~nm}$. Sink conditions were maintained with the addition of an equal volume of fresh medium at the same temperature. The percentage of drug released was expressed with respect to the drug content of the nanoparticles.

\section{In-vivo toxicity studies}

The animal experiments were carried out by following experimental protocol guidelines of council for the purpose of control and supervision of experiments on animals (CPCSEA), Government of India. Experiments were conducted after ethical clearance by the Institutional animal ethical committee (IAEC approval no. 1632/PO/Re/S/12/CPCSEA).

\section{Acute-toxicity study}

10 albino mice were taken for the study. Calculated dose was suspended in $5 \mathrm{ml}$ of the vehicle and were given in divided doses at time interval of $2 \mathrm{~h}$, for 15 days followed-up by monitoring of the animals for any occurrence of signs and symptoms of toxicity or mortality.

\section{Sub-acute toxicity}

6 male and 6 female albino mice, were taken for four test groups. The animals were gavage the relevant doses suspended in $0.5 \mathrm{ml}$ of the vehicle, once daily, for a period of 28 days. At the end of the treatment, the animals were bled from the orbital sinus for clinical pathology assessment which included analysis of various hematology parameters like Hemoglobin [Hgb (g/dl)], total red blood cell count (RBC), total white blood cell count (WBC), packed cell volume (PCV \%) etc. The animals were sacrificed by cervical dislocation. 


\section{RERULTS AND DISCUSSION}

\section{Identification and characterization of Curcumin \\ Fourier transform infrared spectrum (FTIR)}

The drug, polymer, FA-CUR-GNP and CUR-GNP were identified by IR spectroscopy (Fig. 1) and characteristic peaks obtained were compared with standard spectra of pure drug, polymer, folic acid conjugation and non-conjugation as reported in official monograph and were found in agreement with the standard

Functional group frequencies for Curcumin shows (Carbonyl $1651.46 \mathrm{~cm}^{-1}$, Alkenes $1649.8 \mathrm{~cm}^{-1}$, Alcohol 1025.92 $\mathrm{cm}^{-1}$ and Benzene $1431.91 \mathrm{~cm}^{-1}$ ); Gliadin (Amides $1676.84 \mathrm{~cm}^{-}$, Benzene $1559.92 \mathrm{~cm}^{-}$) Folic acid conjugation (carbonyl 1651.46 $\mathrm{cm}^{-}{ }^{1}$,hydroxyl $1225.92 \mathrm{~cm}^{-}{ }^{1}, \quad$ amino3000.69 $\left.\mathrm{cm}^{-}{ }^{1}\right)$; Folic acid non conjugation (phenolic $1227.42 \mathrm{~cm}^{-1}$ ) indicating the purity of drug.

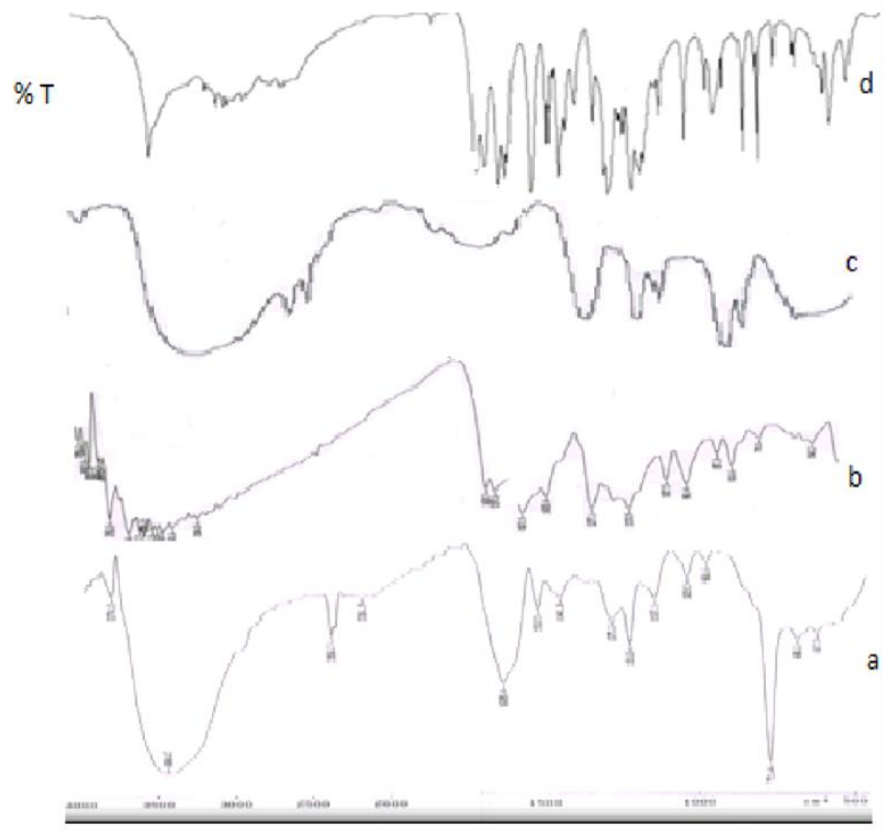

Wave Number $\left(\mathrm{cm}^{-1}\right)$

Fig. 1: FTIR spectra of curcumin, gliadin, FA-CUR-GNP and CUR-GNP

\section{Mass spectroscopy}

The mass spectrum (Fig. 2A and 2B) clearly indicates the exact molecular weight result of Curcumin and Gliadin were of 369.1323 and 542.3 respectively as shown in base peak. The mass spectrum result of Gliadin (Fig. 3) clearly indicates the exact molecular weight 542.3 compared with the standards which was reported in the literature (Jasim and Ali, 1988).

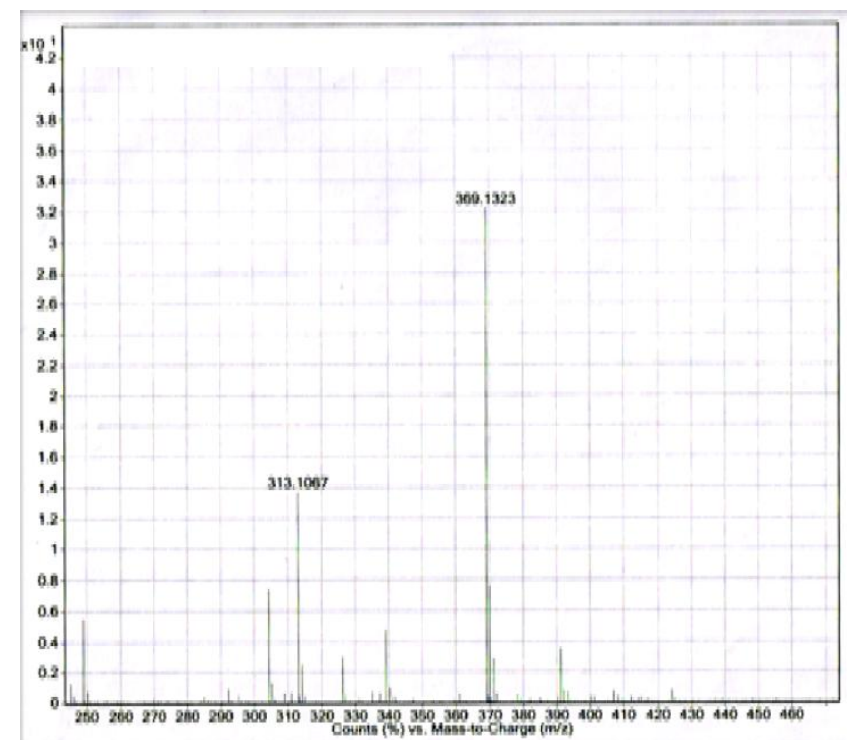

Fig. 2a: Mass spectrum of curcumin.

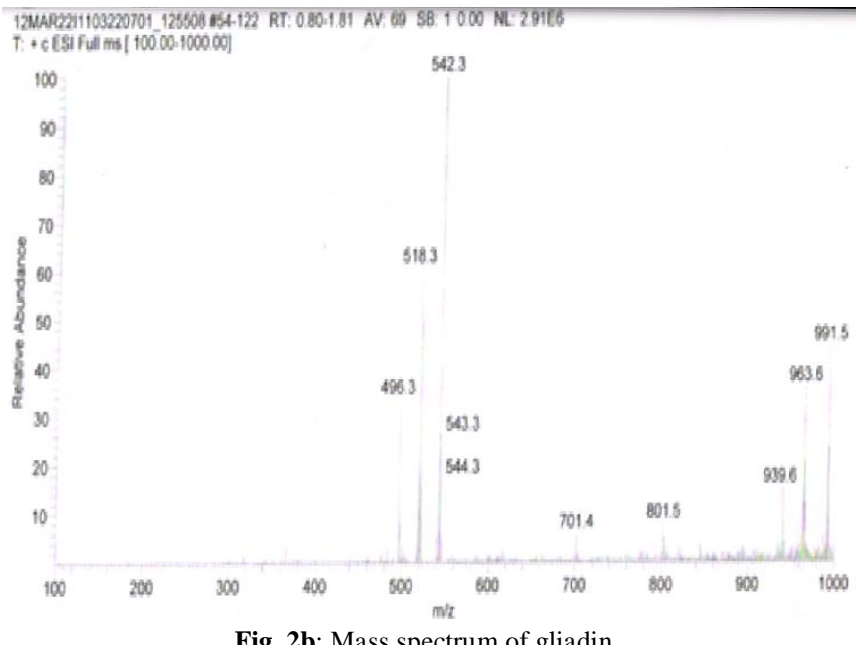

Fig. 2b: Mass spectrum of gliadin.

\section{NMR spectrum of curcumin}

The NMR spectra were recorded on a Bruker WM-600 $(600 \mathrm{MHz})$ spectrometer. The data was processed using the Topspin software (Fig. 3).

The ${ }^{1} \mathrm{H}$ NMR spectrum of curcumin contains two singlets at 3.83 and $9.69 \mathrm{ppm}$ due to the protons of the two methoxy groups and the protons of the two hydroxyl groups respectively which reflects its symmetric structure. The most important signals which give aid to elucidate the molecular structure of the compounds are those arise within the ranges 2.40-2.63, 2.80-3.08 and 4.66-5.08 ppm each of them has the integral of one proton (Wolinski et al., 1990). 


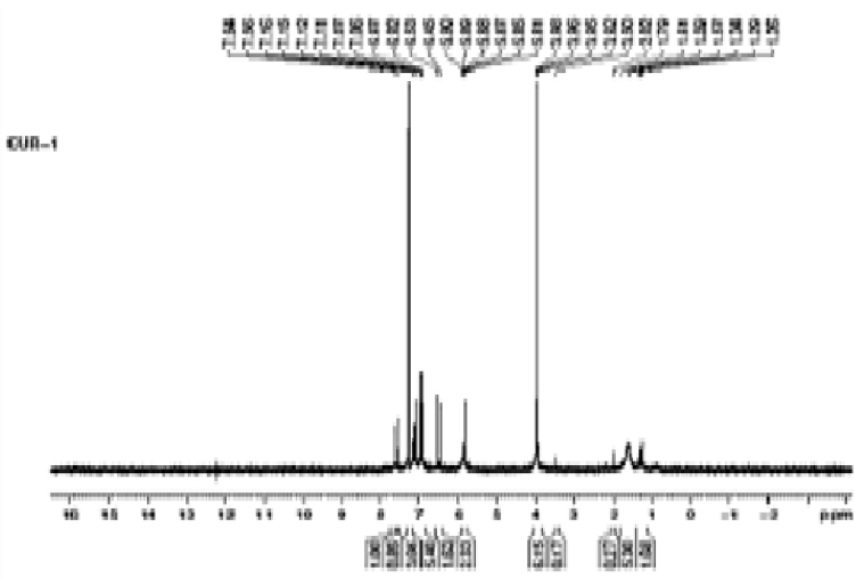

Fig. 3: NMR spectrum of curcumin.

\section{Solubility studies}

\section{Solubility profile of curcumin}

The solubility of curcumin were determined in aqueous and non-aqueous solvents. Curcumin is insoluble in water, slightly soluble in methanol, ethanol, soybean oil, oleic acid and vitamin E acetate but freely soluble in acetone.

\section{Solubility profile of gliadin} $(70 \% \mathrm{v} / \mathrm{v})$

The highest solubility of Gliadin was found in ethanol

\section{Partition coefficient}

The partition coefficient was found to be 3.62 in $n$ octanol: water system. This concludes that drug is lipophilic in nature since the drug did not show any preference to the water and only soluble in the organic solvents. The octanol/water partition coefficient $\left(\log \mathrm{P}_{\mathrm{O} / \mathrm{W}}\right)$ constitutes a quantitative and easily accessible, hydrophobicity measurement. Log $\mathrm{P}$ values are widely used in bio-accumulation studies, in drug absorption and toxicity predictions and lipophilicity of the drug. Partition coefficient values of 3.72 indicates the lipophilic chacteristic of the drug.

\section{Entrapment efficiency}

Size Distribution of various formulation of different batches were presented (Table 1). The entrapment efficiency of F5 and F6 were highest $(52 \%$ and $56 \%$ ) among CUR-GNP and FACUR GNPs respectively. Among the six formulation, F-3 and F-4 were selected as an optimized formulation due to its narrow size distribution as well as good entrapment efficiency and were further evaluated for drug release and in vivo uptake.

\section{Preparation of nanoparticles}

Preparation of folic acid conjugated Gliadin nanoparticles of curcumin were successfully formulated. FACUR-GNPs and CUR-GNPs were carefully envisaged. Folic acid is also conjugated with the polymer that helps in the targeted delivery of the drug towards the cancer cells since many cancer cells are expressing folic acid on its surface. Folic acid has received enormous consideration for biocompatibility or no immunogenicity, high stability and economic cost (Bhattacharya et al., 2011). By mean of receptor-mediated endocytosis, it has been reported that folate conjugates mediate faster internalization kinetics than other approaches through cellular membrane.

\section{Characterization of nanoparticles \\ Particle morphology by TEM}

The particle morphology by TEM (Fig. 4) for both F-3 and F-4 were found to be spherical. The characterization of FACUR-GNPs and CUR-GNPs nanoparticles obtained were of size range of 160-190 nm (Fig. 4). The folic acid conjugated crosslinked particles showed a round morphology with increased size. Transmission electron microscopy has been the preferred method of studying the cellular uptake of nanoparticles. Due to its high resolution TEM enables the imaging of membrane invaginations, vesicle formation and organelles (Song et al., 2010).

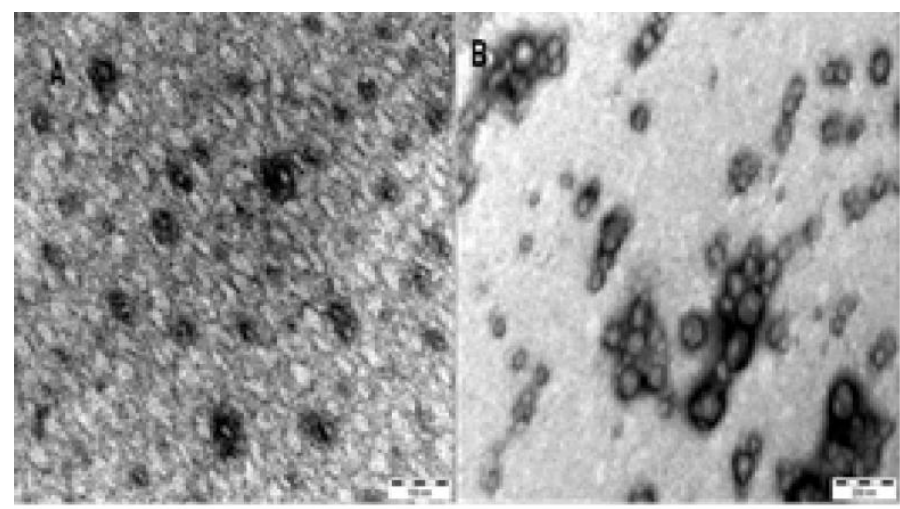

Fig. 4: Transmission electron micrographs of (A) CUR-GNP; (B) FA-CURGNP.

\section{Particle size distribution}

Particle size distribution of F-1, F-2, F-3, F-4, F-5 and F6 were measured (Table 1) and shows that the particle size of CUR-GNP $_{\mathrm{S}}$ is smaller than FA-CUR-GNP $. \quad F-3$ and F-4 formulations of CUR-GNP and FA-CUR-GNPs respectively were found satisfactory. Increased particle size (118.5 to $476.8 \mathrm{~nm}$ ) was due to rise in polymer amount in ethanol (20 to $25 \mathrm{mg}$ ). Firstly modification of CUR and FA onto the surface of $\mathrm{GNP}_{\mathrm{S}}$ increases the particle size secondly, the amine and carboxyl group of gliadin and FA attract counter ions from the solution to form electrical double layer. Increase in particle size could be significantly affected by the polymer amount when other formulation variables were kept constant.

Optimal particle size should be needed for a drug delivery, so that it will not easily leak out of the capillaries and up taken by the macrophages. We developed particle $(190 \mathrm{~nm})$ which is optimal for the cellular uptake and FA conjugation will help in the active targeted delivery of nanoparticle into the cancer site leads to faster accumulation and release of drug to that particular site (Khare and Peppas, 1995). 
Table 1: Particle size optimization.

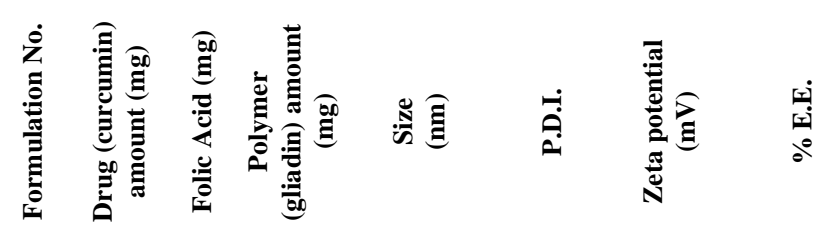

\begin{tabular}{cccccccc}
\hline F-1 & 5 & 5 & 10 & $118.5 \pm 13.7$ & 0.432 & -1.58 & $18 \pm 0.9$ \\
F-3 & 10 & 5 & 20 & $168.1 \pm 13.6$ & 0.339 & -16.5 & $45 \pm 2.6$ \\
F-5 & 12.5 & 5 & 25 & $367.7 \pm 22.8$ & 0.440 & -12.9 & $52 \pm 3.1$ \\
F-2 & 5 & - & 10 & $202.2 \pm 16.2$ & 0.469 & -5.45 & $25 \pm 1.2$ \\
F-4 & 10 & - & 20 & $195.7 \pm 16.5$ & 0.456 & -24.4 & $48 \pm 2.4$ \\
F-6 & 12.5 & - & 25 & $476.8 \pm 25.3$ & 0.392 & -2.43 & $56 \pm 3.5$ \\
\hline
\end{tabular}

Results are expressed in terms of mean \pm SD $(n=3)$, PDI: Polydispersity index, $\mathrm{nm}$ : nanometer, $\mathrm{mV}$ : millivolt, \% EE: percent entrapment efficiency, SD: standard deviation.

F-1, F-3, F-5 CUR-GNP ; F-2, F-4, F-6 FA-CUR-GNP

CUR-GNP (Folic acid non-conjugated gliadin nanoparticles of curcumin)

FA-CUR-GNP (Folic acid conjugated gliadin nanoparticles of curcumin)

\section{Surface charge (zeta potential) studies}

F-3 and F-4 formulations were found satisfactory because of their higher $(-16.5$ and $-24.4 \mathrm{mV}$ ) zeta potential values as mentioned in classification of colloidal system according to zeta potential distribution (Table 2). Greater the zeta potential, greater the stability. Zeta potential was a reliable indicator in the prediction of stability of particles in liquid medium and the possible interactions with other materials. Previous work had shown that negatively charged lipid vesicles (especially with a charge around $-30 \mathrm{mV}$ ) had good stability and might be optimized for drug delivery (Tan et al., 2010).

Table 2: Classification of colloidal system according to zeta potential distribution

\begin{tabular}{ll}
\hline Zeta potential $(\mathbf{m V})$ & Stability behavior of the colloid \\
\hline from 0 to \pm 5 & Rapid coagulation or flocculation \\
from \pm 10 to \pm 30 & Moderate stability \\
from \pm 30 to \pm 40 & Good stability \\
$\mathrm{mV}:$ millivolt & \\
\hline
\end{tabular}

\section{In-vitro release studies}

The in vitro release profile shows how a delivery system works and gives a valuable insight into its in vivo behavior.

The release of CUR from the Gliadin nanoparticles was relatively faster than that from the FA-GNP nanoparticles. About $33 \%$ and $10 \%$ of CUR were released from the GNP and FA-GNP nanoparticles respectively. But after $12 \mathrm{~h}$, the release of CUR from Gliadin nanoparticle was slower than that of FA-GNP nanoparticle. The drug release data obtained for the selected formulation F-3 and F-4 (Fig. 5) and their cumulative \% release were found $92.92 \%$ and $94 \%$ respectively. The result reveals that the cumulative release of CUR from FA-GNP nanoparticle is more than of GNP nanoparticle. In vitro release profile of CUR appeared to be in different patterns. A burst release in the first period. The rapid initial release involved the diffusion of the bound or adsorbed drug at the surface of the nanoparticles. Release of CUR from the gliadin nanoparticles was relatively faster than that from the FA-GNP nanoparticles; this is due to the lower crosslinking ability of the FA-GNP nanoparticles. After $12 \mathrm{~h}$ the release of CUR from Gliadin nanoparticle was slower than that of FA-GNP nanoparticle that due to degradation of the polymer.

In vitro release study of Formulation F3 and F4

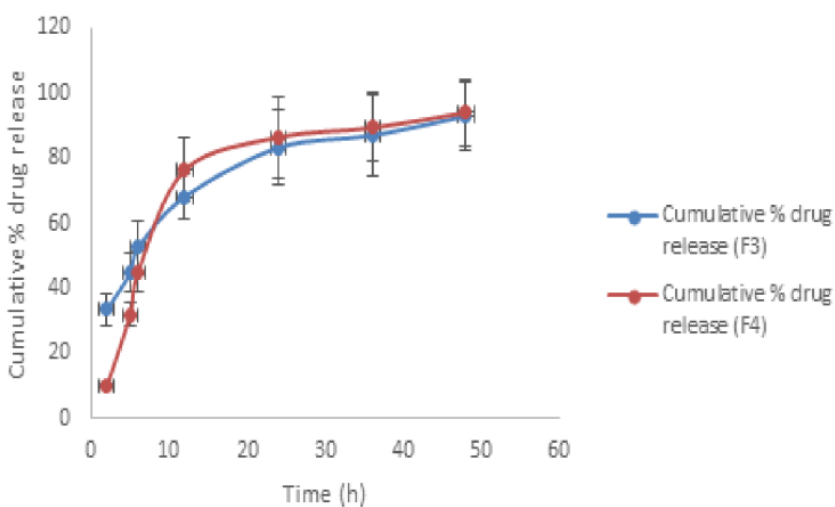

Fig. 5: In vitro release study of formulation F-3 (CUR-GNP) and F-4 (FACUR-GNP).

\section{In-vivo toxicity study}

The toxicity study were performed for both FA-CURGNPs, CUR-GNPs and then compared with the control and toxicity profile was shown in (Fig. 6). The above graph shows that both F-3 and F-4 formulations were safe, compatible and within the normal range. Clinical pathological testing of laboratory animals is a major safety assessment for new drugs in development for general health screening of animals considered for placement in toxicity studies (Leissing et al., 1985). Phosphate-buffered saline ( $\mathrm{pH}$ 6.8) has been used as a vehicle for in vivo exposure and does not alter the biological activity of the test nanoparticle (Sager et al., 2007).

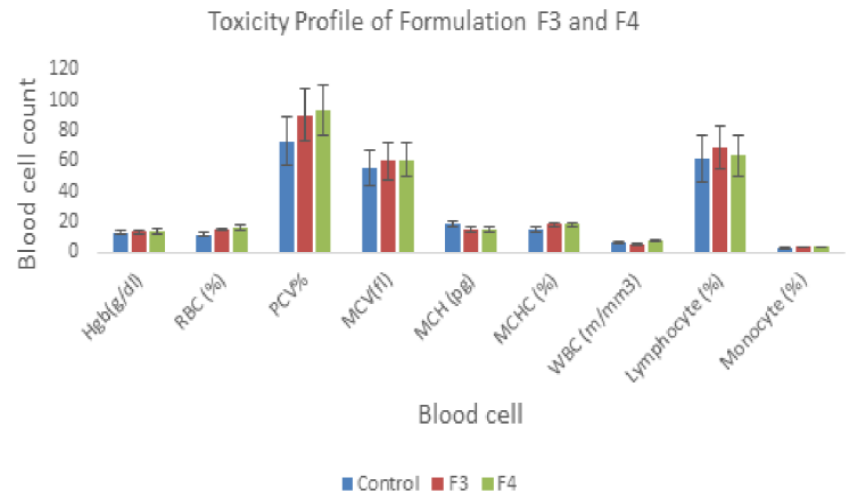

Fig. 6: Toxicity Profile of formulation F-3 (CUR-GNP) and F-4 (FA-CURGNP).

\section{CONCLUSION}

Colon cancer is the third most diagnosed and leading cause of death. The development of multidrug resistance in cancer 
therapeutics results in a minimal response to conventional cytotoxic agents and targeted biological therapies. A great need for alternative treatment options of the disease has encouraged researchers. Curcumin, is a prominent candidate for treating colon cancer. Lower solubility overcome by improving its bioavailability, protect it from degradation and metabolism, and increase its targeting capacity toward cancer tumor(s). Therefore the development of efficient curcumin-gliadin- folate conjugate system is a current challenge in this era. The FA-CUR-GNP showed high in vitro drug release and the stability of the formulations was also maintained. The globule size was also found in the Nano-range, hence providing better bioavailability of the drug. The surface charge was also found to be in range thus, the stability of the formulations was also maintained throughout the experiment. Further in vivo toxicity study also revealed that the formulation was non-toxic.

\section{ACKNOWLEDGEMENTS}

All the authors acknowledge the support of Sigma Aldrich Corp., Switzerland for providing curcumin, gliadin, pluronic F-68 and folic acid as gift samples.

\section{Financial support and sponsorship: Nil.}

Conflict of Interests: There are no conflicts of interest.

\section{REREFERNCES}

Anand $\mathrm{P}$, Sundaram C, Jhurani S, Kunnumakkara AB, Aggarwal BB. Curcumin and cancer: an "old-age" disease with an "ageold" solution. Cancer Lett, 2008; 267:133-164.

Bansal SS, Goel M, Aqil F, Vadhanam MV, Gupta RC. Advanced drug delivery systems of curcumin for cancer chemoprevention. Cancer Prev Res, 2011; 4: 1158-1171.

Bhattacharya D, Das M, Mishra D, Banerjee I, Sahu SK, Maiti TK, Pramanik P. Folate receptor targeted, carboxymethyl chitosan functionalized iron oxide nanoparticles: a novel ultradispersed nanoconjugates for bimodal imaging. Nanoscale, 2011; 3: 1653-1662.

Cho JW, Lee KS, Kim CW. Curcumin attenuates the expression of IL-1beta, IL-6, and TNF-alpha as well as cyclin E in TNF-alpha-treated $\mathrm{HaCaT}$ cells; NfkappaB and MAPKs as potential upstream targets. Int $\mathbf{J}$ Mol Med, 2007; 19: 469-474.

Coester CJ, Langer K, Van Briesen H, Kreuter J. Gelatin nanoparticles by two step desolvation a new preparation method, surface modifications and cell uptake. J Microencapsul, 2000; 17: 187-193.

Duthie SJ. Folic acid deficiency and cancer: mechanisms of DNA instability. Br Med Bull, 1999; 55: 578-592.

Gelperina S, Kisich K, Iseman MD, Heifets L. The potential advantages of nanoparticle drug delivery systems in chemotherapy of tuberculosis. Am J Respir Crit Care Med, 2005; 172: 1487-1490.

Huang MT, Lysz T, Ferraro T , Abidi TF, Laskin JD, Conney $\mathrm{AH}$. Inhibitory effects of curcumin on in vitro lipoxygenase and cyclooxygenase activities in mouse epidermis. Cancer Res, 1991; 51: 813819.

Jahanshahi M, Babaei Z. Protein nanoparticle: a unique system as drug delivery vehicles. Afr J Biotechnol, 2008; 7: 4926-4934.

Jasim F, Ali F. A novel method for the spectrophotometric determination of curcumin and its application to curcumin spices. Microchem J, 1988; 38: 106-110.

Jobin C, Bradham CA, Russo MP, Juma B, Narula AS, Brenner DA, Sartor RB. Curcumin blocks cytokine-mediated NF-kappa B activation and proinflammatory gene expression by inhibiting inhibitory factor I-kappa B kinase activity. J Immunol, 1999; 163: 3474-3483.

Khare AR, Peppas NA. Swelling/deswelling of anionic copolymer gels. Biomaterials, 1995, 16:559-567.

Leissing N, Izzo R, Sargent $H$. Variance estimates and individuality ratios of 25 serum constituents in beagles. Clin Chem, 1985; 31:83-86.

Liu JY, Lin SJ, Lin JK. Inhibitory effects of curcumin on protein kinase $\mathrm{C}$ activity induced by 12-O-tetradecanoyl-phorbol-13acetate in NIH 3 T3 cells. Carcinogenesis, 1993; 14: 857-861.

Moradhaseli S, Mirakabadi AZ, Dounighi NM, Soheily S, Borumand MR. Preparation and characterization of sodium alginate nanoparticles containing ICD-85 (venom derived peptides). Int J Innov Appl Studies, 2013; 4: 534-542.

Pisani P, Bray F, Parkin DM. Estimates of the world-wide prevalence of cancer for 25 sites in the adult population. Int $\mathrm{J}$ Cancer, 2002; 97: 72-81.

Sager TM, Porter DW, Robinson VA, Lindsley WG, Schwegler-Berry DE, Castranova V. Improved method to disperse nanoparticles for in vitro and in vivo investigation of toxicity. Nanotoxicology, 2007; 1:118-129.

Seyfried TN, Shelton LM. Cancer as a metabolic disease. Nutr Metab, 2010; 7:7.

Song MM, Song WJ, Bi H, Wang J, Wu WL, Sun J, Yu M. Cytotoxicity and cellular uptake of iron nanowires. Biomaterials, 2010; 31:1509-1517.

Srimal RC, Dhawan BN. Pharmacology of diferuloyl methane (curcumin), a non-steroidal anti-inflammatory agent. J Pharm Pharmacol, 1973; 25: 447-452.

Storp Bv, Engel A, Boeker A, Ploeger M, Langer K. Albumin nanoparticles with predictable size by desolvation procedure. J Microencapsul, 2012; 29: 138-146.

Tan QY, Wang N, Yang H, Zhang LK, Liu S, Chen L, Liu J, Zhang L, Hu NN, Zhao CJ, Zhang JQ. Characterization, stabilization and activity of uricase loaded in lipid vesicles. Int J Pharm, 2010; 384:165172.

Tuttle S, Hertan L, Daurio N, Porter S, Kaushick C, Li D, Myamoto S, Lin A, O'Malley BW, Koumenis C.The chemopreventive and clinically used agent curcumin sensitizes HPV-but not HPV+ HNSCC to ionizing radiation, in vitro and in a mouse orthotopic model. Cancer Biol Ther, 2012; 13: 575-584.

Weber C, Coester C, Kreuter J, Langer K. Desolvation process and surface characterization of protein nanoparticles. Int J Pharm, 2000; 194: 91-102.

Wolinski K, Hinton JF, Pulay P. Efficient implementation of the gauge-independent atomic orbital method for NMR chemical shift calculations. J Am Chem Soc, 1990, 112: 8251-8260.

Xiang G, Wu J, Lu Y, Liu Z, Lee RJ. Synthesis and evaluation of a novel ligand for folate-mediated targeting liposomes. Int J Pharm, 2008; 356: 29-36.

Yanyu X, Yunmei S, Zhipeng C, Qineng P. The preparation of silybin-phospholipid complex and the study on its pharmacokinetics in rats. Int J Pharm, 2006; 307:77-82.

\section{How to cite this article:}

Sonekar S, Mishra MK, Patel AK, Nair S, Singh CS, Singh AK. Formulation and evaluation of Folic acid conjugated gliadin nanoparticles of curcumin for targeting colon cancer cells. J App Pharm Sci, 2016; 6 (10): 068-074. 